Document downloaded from:

http://hdl.handle.net/10251/67812

This paper must be cited as:

Orellana, M.; Selga, J.; Sans, M.; Rodríguez Pérez, AM.; Boria Esbert, VE.; Martín Antolín, JF. (2015). Synthesis of slow-wave structures based on capacitive-loaded lines through aggressive space mapping (ASM). International Journal of RF and Microwave ComputerAided Engineering. 25(7):629-638. doi:10.1002/mmce.20901.

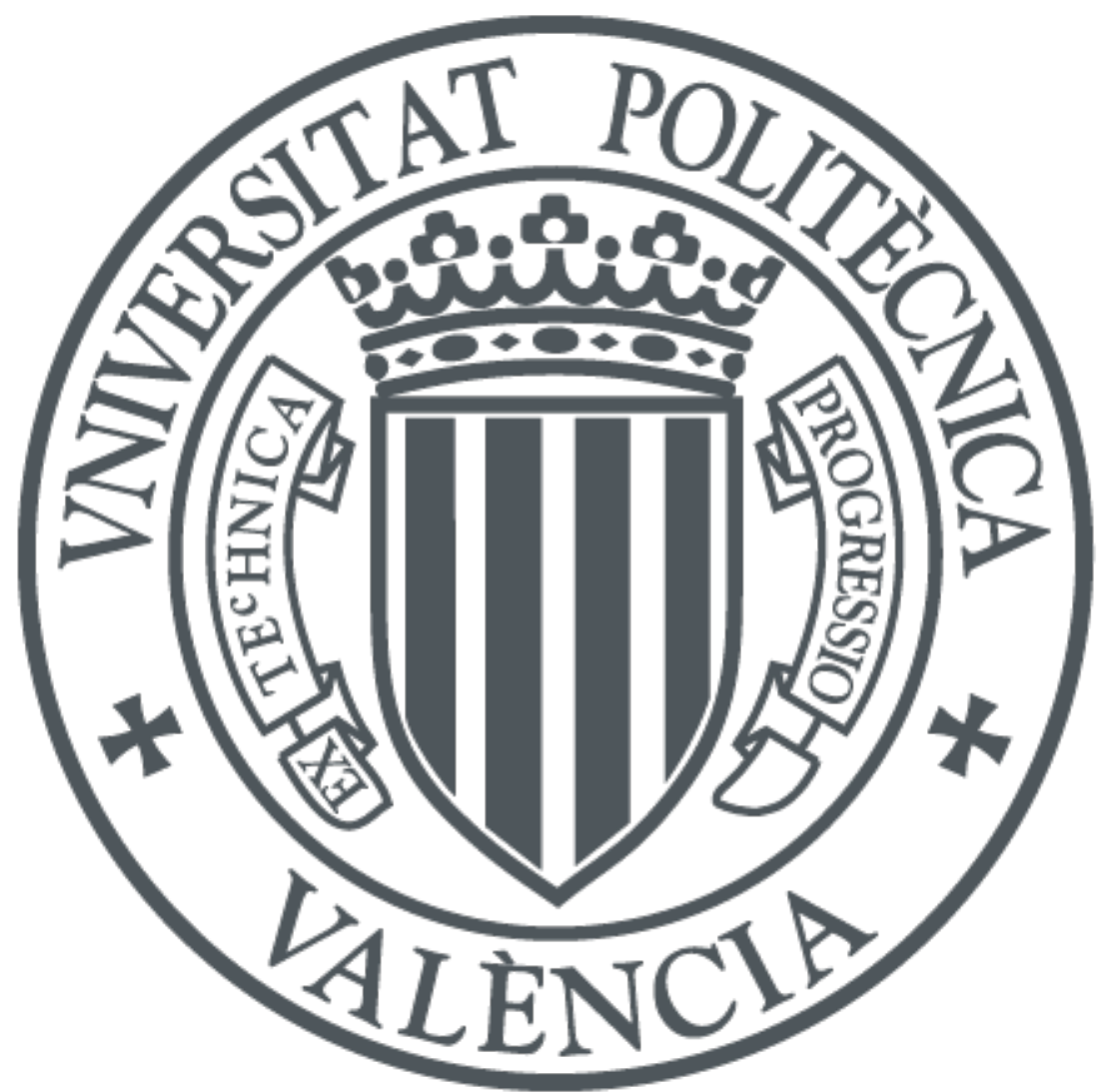

The final publication is available at

http://dx.doi.org/10.1002/mmce.20901

Copyright Wiley

Additional Information 


\title{
Synthesis of Slow-Wave Structures Based on Capacitive-Loaded Lines through Aggressive Space Mapping (ASM)
}

\author{
${ }^{1}$ Marco Orellana, ${ }^{1}$ Jordi Selga, ${ }^{1}$ Marc Sans, ${ }^{2}$ Ana Rodríguez, ${ }^{2}$ Vicente Boria, ${ }^{1}$ Ferran \\ Martín \\ ${ }^{1}$ GEMMA/CIMITEC, Departament d'Enginyeria Electrònica, Universitat Autònoma de \\ Barcelona, 08193 Bellaterra, Spain. E-mail: Ferran.Martin@uab.es \\ ${ }^{2}$ Departamento de Comunicaciones-iTEAM, Universidad Politécnica de Valencia, \\ 46022 Valencia, Spain. E-mail: vboria@dcom.upv.es
}

\begin{abstract}
This paper is focused on the automated synthesis of slow-wave structures based on microstrip lines loaded with patch capacitors. Thanks to the presence of the shunt capacitors, the effective capacitance of the line is enhanced, and the phase velocity of the structure can be made significantly smaller than the one of the unloaded line. The target is to achieve the layout of the slow-wave structure able to provide the required slow-wave ratio, characteristic (Bloch) impedance and electrical length (i.e., the usual specifications in the design of slow-wave transmission lines). To this end, a two-step synthesis method, based on the aggressive space mapping (ASM) algorithm, is proposed for the first time. Through the first ASM algorithm, the circuit schematic providing the target specifications is determined. Then, the second ASM optimizer is used to generate the layout of the structure. In order to illustrate the potential of the proposed synthesis method, two application examples are successfully reported. The two-step ASM algorithm is able to provide the layout of the considered structures from the required specifications, without the need of an external aid in the process.
\end{abstract}

Keywords: space mapping, slow-wave transmission lines, microwave circuits. 


\section{Introduction}

Slow-wave transmission lines are wave guiding structures exhibiting a phase velocity smaller than the one corresponding to ordinary lines implemented in the same substrate. Owing to the slow-wave behavior of these lines, the wavelength is also small, as compared to ordinary lines, and hence the circuit size can be significantly reduced (see the recent review on artificial transmission lines [1], that includes slow-wave transmission lines). Typically, slow-wave transmission lines are implemented by periodically loading a host line with shunt connected capacitors, either lumped or semi-lumped (e.g., patch capacitors). By means of this capacitive loading, the effective capacitance of the line is enhanced and the phase velocity is reduced. Slow-wave transmission lines have found applications in microwave circuit miniaturization [24]. However, due to periodicity, these structures exhibit stop bands, which are of interest for spurious suppression. Thus, slow-wave structures have been applied to the design of microwave circuits with reduced size and spurious suppression $[5,6]$.

Slow-wave transmission lines implemented by means of capacitively loaded lines are relatively simple structures. However, in order to satisfy the usual specifications for circuit design, namely, characteristic impedance, electrical length, and slow-wave ratio (i.e., the ratio between the phase velocity of the loaded and unloaded line), optimization is typically necessary. In this paper, we propose an automated synthesis process for capacitively-loaded slow-wave transmission lines based on aggressive space mapping (ASM) [7-9]. This synthesis technique has been applied to the design of many planar microwave circuits [10-14]. Recently, we have proposed a two-step ASM technique useful for microwave circuits described by a circuit schematic, where the optimum schematic (i.e., the one that satisfies specifications) is first determined (first step), and then the circuit layout is automatically generated (second step) [15, 16]. This two-step ASM technique has been successfully applied to the design of bandpass filters based on stepped impedance resonators and shunt stubs coupled through admittance inverters $[15,16]$. Similarly, we have implemented a two-step ASM algorithm for the synthesis of slow-wave transmission lines. Through the first algorithm, the optimum schematic, consisting on a cascade of transmission line sections (host line) and shunt capacitors, is determined. Then, from the optimum capacitance value and host line characteristics (impedance and electrical length), the layout is automatically generated.

The work is organized as follows. In the second section, the lumped element equivalent circuit model of the considered slow-wave structures, and the main design formulas, are provided. Section 3 is focused on the general formulation of ASM. Section 4 is devoted to the description of the new proposed two-step ASM synthesis algorithm. Finally, two illustrative synthesis examples of slow wave transmission lines are reported in section 5. The conclusions are highlighted in section 6 .

\section{Slow-wave structures based on capacitively loaded lines}

The schematic of the slow-wave structures considered in this work is depicted in Fig. 1(a). The host line, with phase constant $k$ (electrical length of the unit cell $k l$ ), and characteristic impedance $Z_{o}$, is periodically loaded with shunt connected capacitances, $C_{l s}$. From now on, we will refer to $k$ and $Z_{o}$ as the phase constant and characteristic impedance of the unloaded line, respectively, in order to distinguish such variables from the corresponding characteristic impedance (actually Bloch impedance), $Z_{B}$, and phase constant, $\beta$, of the capacitively-loaded lines. The dispersion relation and Bloch impedance of the structure of Fig. 1 can be obtained 
from the ABCD matrix of the unit cell, following standard Bloch wave analysis of periodic structures [17]. In particular, the dispersion relation is given by:

$$
\cos \beta l=\cos k l-\frac{\omega C_{l s} Z_{o}}{2} \sin k l
$$

The Bloch impedance is given by:

and the slow-wave ratio is given by:

$$
Z_{B}=\frac{Z_{0}\left[\sin k l-Z_{0} \omega C_{l s} \sin ^{2}(k l / 2)\right]}{\sqrt{1-\left(\cos k l-\frac{1}{2} Z_{0} \omega C_{l s} \sin k l\right)^{2}}}
$$

$$
s w r=\frac{v_{p}}{v_{p o}}=\frac{\omega / \beta}{\omega / k}=\frac{k l}{\beta l}
$$

The previous expressions are cumbersome and, typically, the lumped element equivalent circuit model is invoked for design purposes. It is depicted in Fig. 1(b), where $C$ and $L$ are the persection capacitance and inductance of the line. According to this model, the Bloch impedance, the electrical length (unit cell) and the slow wave ratio are given by:

$$
\begin{gathered}
Z_{B}=\sqrt{\frac{L}{C+C_{l s}}} \\
\phi_{\text {Cell }}=\beta l=\omega \sqrt{L\left(C+C_{l s}\right)} \\
s w r=\frac{v_{p}}{v_{p o}}=\frac{l / \sqrt{L\left(C+C_{l s}\right)}}{l / \sqrt{L C}}=\frac{1}{\sqrt{1+\frac{C_{l s}}{C}}}
\end{gathered}
$$

respectively. From the previous expressions, the elements of the circuit of Fig. 1(b) can be easily isolated. However, the validity of the model of Fig. 1(b) is restricted to large values of $C_{l s}$ as compared to $C$. Indeed, if the condition $C_{l s}>C$ is satisfied, the agreement between the lumped element model and the schematic is good up to the cut-off frequency (at low frequencies, the model is valid regardless of the relative values between $C$ and $C_{l s}$ ). However, this condition $\left(C_{l s}>>C\right)$ is not always satisfied. This justifies the first ASM proposed in this work, where, from the element values provided by isolation of expressions (4)-(6), we determine the optimum schematic of Fig. 1(a), namely, the one that satisfies the target specifications. An alternative to this ASM process would be the numerical solution of expressions (1)-(3), but we have opted for implementing an ASM algorithm, which is simple, fast and accurate. Indeed, the first ASM provides a technique for the numerical solution of expressions (1)-(3). 

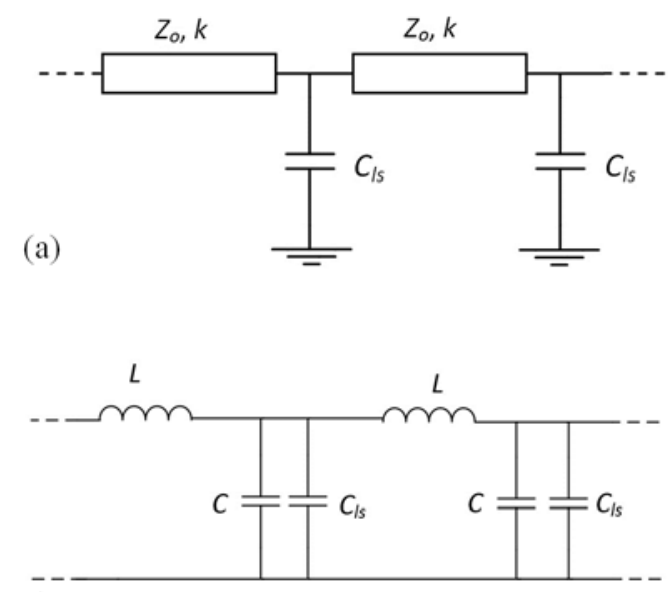

(b)

Fig. 1. Schematic of the slow-wave structure based on a capacitively loaded line (a), and lumped element equivalent circuit model (b).

\section{General formulation of aggressive space mapping (ASM)}

Space mapping (SM) is a technique extensively used for the synthesis and optimization of microwave components. It uses two simulation spaces [7]-[9]: (i) the optimization space, $\mathbf{X}_{\mathbf{c}}$, where the variables are linked to a coarse model, which is simple and computationally efficient, although not accurate, and (ii) the validation space, $\mathbf{X}_{\mathbf{f}}$, where the variables are linked to a fine model, typically more complex and CPU intensive, but significantly more precise. In each space, a vector containing the different model parameters can be defined. Let us call such vectors $\mathbf{x}_{\mathbf{f}}$ and $\mathbf{x}_{\mathbf{c}}$ in the fine and coarse model spaces, respectively, and let us designate by $\mathbf{R}_{\mathbf{f}}\left(\mathbf{x}_{\mathbf{f}}\right)$ and $\mathbf{R}_{\mathbf{c}}\left(\mathbf{x}_{\mathbf{c}}\right)$ their corresponding responses. The key idea behind any SM optimization process is to generate an appropriate parameter transformation

$$
\mathbf{x}_{\mathbf{c}}=\mathbf{P}\left(\mathbf{x}_{\mathbf{f}}\right)
$$

mapping the fine model parameter space to the coarse model parameter space such that

$$
\left\|\mathbf{R}_{\mathbf{f}}\left(\mathbf{x}_{\mathbf{f}}\right)-\mathbf{R}_{\mathbf{c}}\left(\mathbf{x}_{\mathbf{c}}\right)\right\| \leq \eta
$$

in some predefined region, $\|\cdot\|$ being a certain suitable norm and $\eta$ a small positive number close to zero. If $\mathbf{P}$ is invertible, then the inverse transformation:

$$
\mathbf{x}_{\mathbf{f}}=\mathbf{P}^{-1}\left(\mathbf{x}_{\mathrm{c}}^{*}\right)
$$

is used to find the fine model solution, which is the image of the coarse model solution, $\mathbf{x}_{\mathbf{c}}{ }^{*}$, that gives the target response, $\mathbf{R}_{\mathbf{c}}\left(\mathbf{x}_{\mathbf{c}}{ }^{*}\right)$.

The determination of $\mathbf{P}$ according to the procedure reported in [7] follows an iterative process that is rather inefficient. The efficiency of the method was improved by introducing a quasiNewton type iteration [8], resulting in a faster convergence, and giving rise to the so-called aggressive space mapping (ASM) [8]. The goal in ASM is to minimize the following error function:

$$
\mathbf{f}\left(\mathbf{x}_{\mathbf{f}}\right)=\mathbf{P}\left(\mathbf{x}_{\mathbf{f}}\right)-\mathbf{x}_{\mathbf{c}}^{*}
$$


Let us assume that $\mathbf{x}_{\mathbf{f}}^{(j)}$ is the $j$-th approximation to the solution in the validation space, and $\mathbf{f}^{(j)}$ the error function corresponding to $\mathbf{f}\left(\mathbf{x}_{\mathbf{f}}^{(j)}\right)$. The next vector of the iterative process $\mathbf{x}_{\mathbf{f}}{ }^{\left(j^{+} 1\right)}$ is obtained by a quasi-Newton iteration according to

$$
\mathbf{x}_{\mathbf{f}}^{(j+1)}=\mathbf{x}_{\mathbf{f}}^{(j)}+\mathbf{h}^{(j)}
$$

where $\mathbf{h}^{(j)}$ is given by:

$$
\mathbf{h}^{(j)}=-\left(\mathbf{B}^{(j)}\right)^{-1} \mathbf{f}^{(j)}
$$

and $\mathbf{B}^{(j)}$ is an approach to the Jacobian matrix which is also updated by a simplification of the classical Broyden formula $[8,18]$ at each iterative step.

$$
\mathbf{B}^{(j+1)}=\mathbf{B}^{(j)}+\frac{\mathbf{f}^{(j+1)} \mathbf{h}^{(j) T}}{\mathbf{h}^{(j) T} \mathbf{h}^{(j)}}
$$

In (13), $\mathbf{f}^{(j+1)}$ is obtained by evaluating (10) using a certain parameter extraction method providing the coarse model parameters from the fine model parameters, and the super-index $T$ stands for transpose.

The implementation of the ASM algorithm is well reported in [8]. In the next section, the new proposed two-step ASM synthesis algorithm is explained in detail.

\section{The proposed two-step ASM synthesis method}

The developed ASM algorithm for the unattended synthesis of slow-wave structures based on capacitively loaded lines is divided in two steps: (i) the determination of the optimum schematic, providing the required specifications, and (ii) the generation of the layout. Let us now describe in detail both ASM stages.

\subsection{First ASM: determination of the optimum schematic}

For the determination of the schematic [given by the circuit of Fig. 1(a)] providing the target specifications, we have developed a specific ASM algorithm. The variables of the coarse space are the three specifications: Bloch impedance, $Z_{B}$, electrical length per unit cell, $\beta$ l, and slowwave ratio, swr. The response of these variables in the coarse space is simply given by the electrical simulation of the circuit of Fig. 1(b) with the element values inferred from expressions (4)-(6). The variables of the fine model are $Z_{o}, k l$ and $C_{l s}$, and the response of this model is given by the circuit simulation of the schematic of Fig. 1(a).

To initiate this first ASM, we must provide the values of the variables of the fine model according to some criterion. The most straightforward one is to identify $C_{l s}$ with the value of this variable inferred by isolation of (4)-(6). The values of $C$ and $L$ also provide $Z_{o}$, i.e.,

$$
Z_{o}=\sqrt{\frac{L}{C}}
$$

and $k l$, given by:

$$
k l=\omega \sqrt{L C}
$$


Once the variables of the fine model are determined, the response is obtained by circuit simulation of the schematic (Fig. 1a). From this response (or by means of expressions 1-3), we extract the variables of the coarse model corresponding to the first iteration $\mathbf{x}_{\mathbf{c}}{ }^{(1)}$. Then, we compare such values with the target $\left(\mathbf{x}_{\mathbf{c}}{ }^{*}\right)$ and this gives the first error function (10). Unless this error function is smaller than a certain predetermined value, the matrix $\mathbf{B}$ must be calculated. To obtain the first approximation to this matrix, we slightly perturb each variable of the fine model from the first value inferred as indicated above. Then we obtain the circuit response, and from it we obtain the resulting variables of the coarse model corresponding to each perturbation. The relative changes can be expressed in a matrix form as follows:

$$
\mathrm{B}^{(1)}=\left(\begin{array}{ccc}
\frac{\delta \mathrm{Z}_{B}}{\delta \mathrm{Z}_{o}} & \frac{\delta \mathrm{Z}_{B}}{\delta k l} & \frac{\delta \mathrm{Z}_{B}}{\delta C_{l s}} \\
\frac{\delta \beta l}{\delta Z_{o}} & \frac{\delta \beta l}{\delta k l} & \frac{\delta \beta l}{\delta C_{l s}} \\
\frac{\delta s w r}{\delta Z_{o}} & \frac{\delta s w r}{\delta k l} & \frac{\delta s w r}{\delta C_{l s}}
\end{array}\right)
$$

which corresponds to the initial matrix $\mathbf{B}$. Once the matrix $\mathbf{B}^{(1)}$ is known, the fine model parameters of the following iteration can be derived from (11), and the process is iterated until convergence is obtained.

\subsection{Second ASM: determination of the layout}

Once the optimum schematic has been obtained, the layout is determined through a second iterative ASM process. The first step is the determination of the side length $\left(l_{p}\right)$ of the capacitive patch corresponding to the shunt capacitor, $C_{l s}$. The geometry is depicted in Fig. 2(a), where a square shaped patch connected to the host line through a wide and short access strip (to avoid any inductive effect) is considered. In this work, the distance between the host line and the patch (length of the access strip, $\left.l_{a c c}\right)$ is set to a fixed value $(0.583 \mathrm{~mm})$, whereas its width $\left(W_{\text {acc }}\right)$ is set to a fraction of the patch dimension $l_{p}$ (specifically, it has been found that $50 \%$ of the patch dimension is a good choice).

To determine $l_{p}$ an independent one variable ASM iterative algorithm has been developed. The variable of the coarse model is the capacitance $C_{l s}$, whereas $l_{p}$ is the variable of the fine model. The first value of $l_{p}$ is inferred from the target value of $C_{l s}$ determined from the first ASM, through the parallel plate capacitor formula. Then the capacitance of this patch is inferred from the electromagnetic simulation by considering the ports indicated in Fig. 2(a), specifically by inferring the admittance of the shunt load [17], and it is compared to the target value. The matrix $\mathbf{B}$, actually composed of a single element, is initiated by varying the patch dimension $l_{p}$ and recording the effects of this variation on the capacitance of the patch. Finally, the process is iterated through (11).

The width of the host line ( $W_{\text {host}}$ ) is not considered to be an optimization variable, since the value provided by well-known microstrip formulas [17] (giving the line width as a function of the characteristic impedance $Z_{o}$ and substrate parameters) are very accurate. The remaining parameter to be determined is the length of the host line, $l$. This is determined through another independent ASM iterative process, where the variable of the coarse model is the electrical length of the loaded line, i.e., $\beta l$, rather than $k l$ (the electrical length of the unloaded line). By 
this means, we correct any phase shift introduced by the capacitive patch. The first value of $l$ is obtained from the optimum value of $k l$ determined at the first ASM. Then, the phase of the whole loaded structure is inferred from electromagnetic simulation, and it is compared to the target value $\beta l$, in order to determine the first error function. The iterative process is then initiated by calculating the first approximation to the Broyden matrix, and optimization of $l$ is done through (11), as indicated before in reference to the one-variable ASM algorithm for the determination of $l_{p}$. The geometry of the unit cell of the slow-wave structure is depicted in Fig. 2(b).

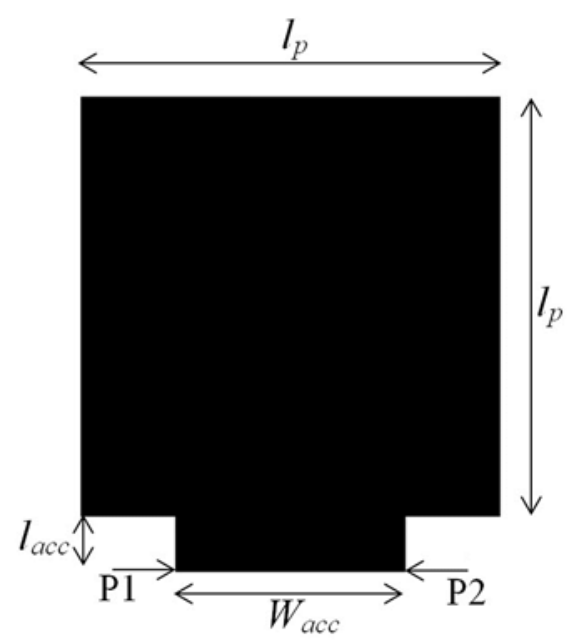

(a)

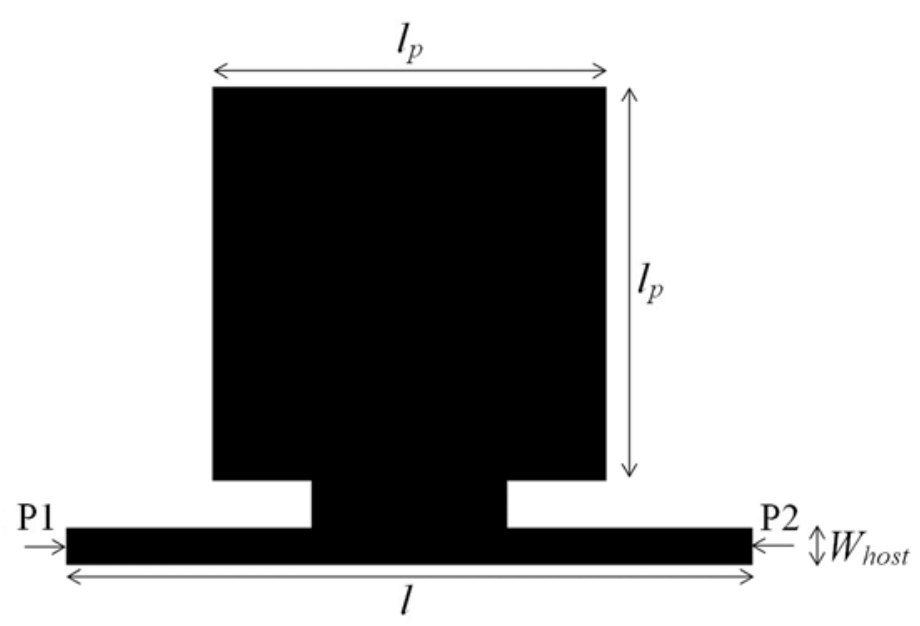

(b)

Fig. 2. Topology and relevant dimensions of the capacitive patch (a) and whole unit cell (b).

For better understanding, the flow diagram of the complete two-step ASM algorithm is depicted in Fig. 3. Using this ASM iterative process, the synthesis of slow-wave structures from specifications can be carried out automatically, following a completely unattended scheme. 


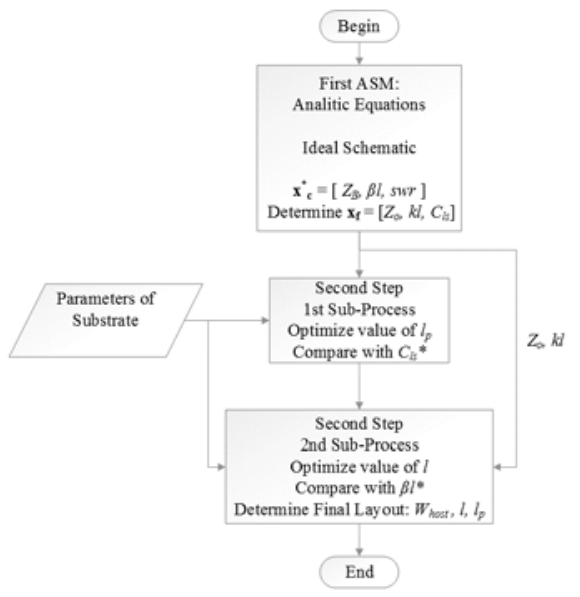

Fig. 3. Flow diagram of the proposed two-step ASM. 


\section{Synthesis examples}

To illustrate the potential of the proposed approach, we have considered the synthesis of two different cells: (1) a cell with electrical length $\phi_{\text {cell }}=\beta l=90^{\circ}$, Bloch impedance $Z_{B}=50 \Omega$, and slow-wave factor $s w r=0.5$, and (2) a cell with electrical length $\phi_{\text {cell }}=\beta l=45^{\circ}$, Bloch impedance $Z_{B}=50 \Omega$, and slow-wave factor $s w r=0.5$. The operating frequency in both cases is $f_{o}=1 \mathrm{GHz}$.

\subsection{Example 1: $\phi_{c e l l}=90^{\circ}, Z_{B}=50 \Omega, s w r=0.5$}

For the considered target specifications, inversion of expressions (4)-(6) provides the following element values for the circuit of Fig. 1(b): $C=1.25 \mathrm{pF}, C_{l s}=3.75 \mathrm{pF}$ and $L=12.5 \mathrm{nH}$. From equations (14)-(15), the values of the first iteration of the fine model, $\mathbf{x}_{\mathbf{f}}{ }^{(1)}$ are: $Z_{o}=100.0 \Omega, k l=$ $45.0^{\circ}$, and $C_{l s}=3.75 \mathrm{pF}$. Using the first ASM algorithm, we have determined the optimum schematic. Convergence, with a relative error of $0.033 \%$, has been achieved after 5 iterations. The resulting values of the fine model are $Z_{o}=120.7 \Omega, k l=45.0^{\circ}$, and $C_{l s}=2.63 \mathrm{pF}$. The response of the schematic (using the previous values of the fine model parameters) is depicted in Fig. 4 (where the insertion/return loss, characteristic impedance and electrical length can be observed). In view of this figure, it is clear that the target specification at the schematic level are satisfied to a very good approximation. i.e., the resulting electrical length, Bloch impedance and slow-wave factor of the cell after convergence are found to be $Z_{B}=50.01 \Omega, \phi_{\text {cell }}=89.99^{\circ}$ and $s w r=0.50$, respectively.

Once the optimum schematic has been obtained, the application of the second ASM has led us to the layout depicted in Fig. 5 (the Rogers RO4003C substrate with dielectric constant $\varepsilon_{r}=3.55$ and thickness $h=0.813 \mathrm{~mm}$, has been considered). The response of this structure, inferred from electromagnetic simulation using the Agilent Momentum commercial simulator, is also depicted in Fig. 4, and reveals that the target specifications are satisfied to a very good approximation at the design frequency, in spite that neither the Bloch impedance nor the slow-wave factor are variables in the optimization process (we have simply optimized patch dimensions and the electrical length of the cell). The second ASM does not involve the optimization of the patch dimensions, length, and width of the line, simultaneously. Rather than this, we have simply optimized the patch dimensions and then the length of the cell, by means of two independent sub-processes with only one optimization variable in each case. The result is a very fast second step ASM algorithm which has been proven to provide accurate results. It is important to mention that optimization has been done at the considered operating frequency. At sufficiently high frequencies (close to the cut-off frequency), discrepancies arise and are due to the fact that distributed effects in the patch capacitance cannot be neglected. Thus, such discrepancies are attributed to a limitation of the model (schematic), where the patch capacitance is described by a lumped element, but not to the proposed optimization algorithm.

It is remarkable that the synthesis algorithm is very efficient, as revealed by the small number of iterations required to achieve the cell layout. The process is completely unattended and the time spent in a computer with Intel ${ }^{\circledR}$ Core ${ }^{\mathrm{TM}}$ i5-3470 Processor is about 3 minutes. It is noteworthy that for each electromagnetic simulation we use a high mesh density to achieve accurate results. 


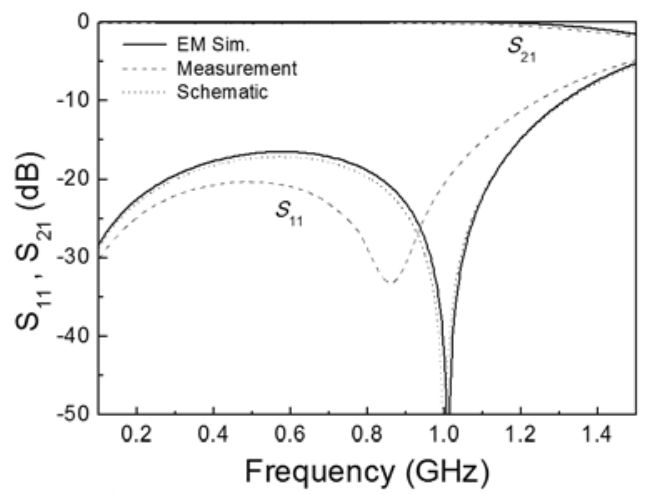

(a)

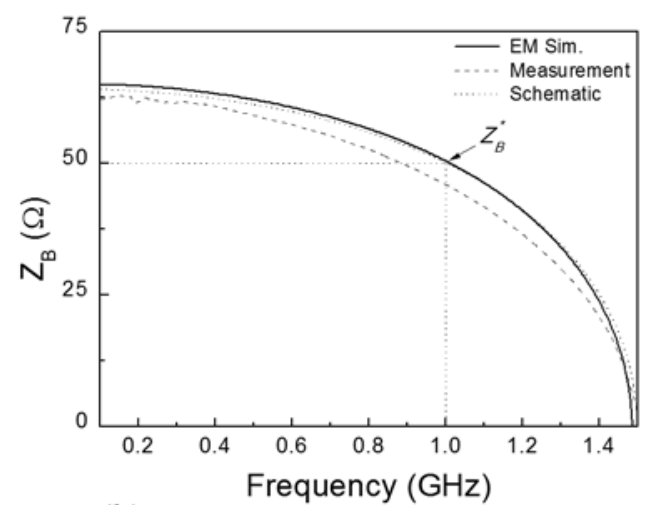

(b)

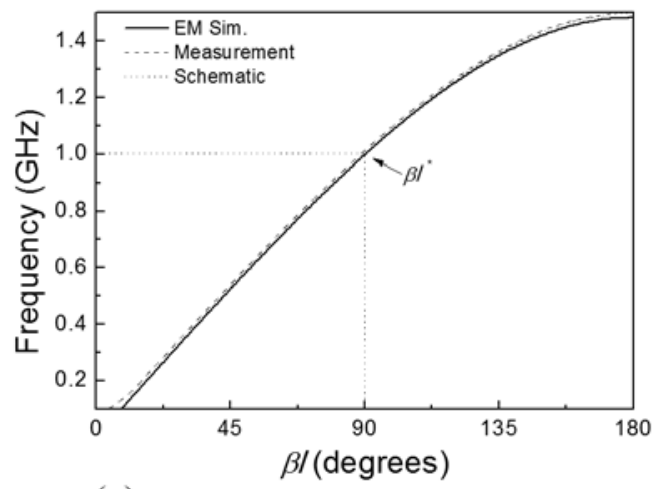

(c)

Fig. 4. Response of the cell considered in example 1. (a) Insertion and return loss, (b) characteristic impedance, and (c) electrical length. 


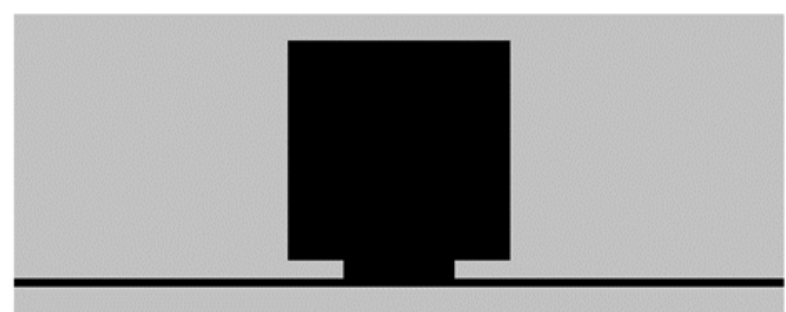

(a)

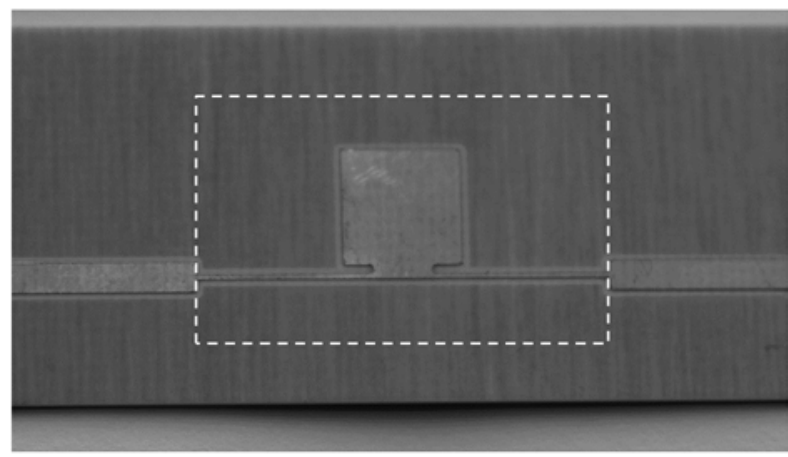

(b)

Fig. 5. Layout (a) and photograph (b) of the synthesized cell of example 1. Dimensions are: $l=25.19$ $\mathrm{mm}, l_{p}=7.23 \mathrm{~mm}, W_{\text {acc }}=3.61 \mathrm{~mm}\left(0.5 l_{p}\right)$, $W_{\text {host }}=0.27 \mathrm{~mm}$

The cell of Fig. 5 has been fabricated by means of the LPKF HF100 drilling machine and has been characterized by means of the Agilent E8364B vector network analyzer and the Anritsu Universal test fixture Model 3680-20. The measured insertion and return loss, as well as the characteristic impedance and electrical length, are also included in Fig. 4. There are some discrepancies attributed to tolerances in cell dimensions and dielectric constant, and also to the effects of the access lines (despite the fact that a de-embedding process has been applied). For most applications, the experimental results reveal that the fabricated structures can be useful since the required specifications, at the operating frequency, are satisfied to a reasonable approximation. Nevertheless, the experimental results are determined by factors (indicated above) external to the described synthesis process, which provides very accurate results (the idea and implementation of the proposed two-step ASM algorithm has been the main focus of this paper and has been demonstrated to be fast and accurate).

\subsection{Example 2: $\phi_{c e l l}=45^{\circ}, Z_{B}=50 \Omega$, swr $=0.5$}

In this case, expressions (4)-(6) give $C=0.625 \mathrm{pF}, C_{l s}=1.875 \mathrm{pF}$ and $L=6.25 \mathrm{nH}$, and from equations (14)-(15) the values of the first iteration of the fine model, $\mathbf{x}_{\mathbf{f}}^{(1)}$ are: $Z_{o}=100.0 \Omega, k l=$ $22.5^{\circ}$, and $C_{l s}=1.875 \mathrm{pF}$. The parameters of the optimum schematic (obtained by means of the first ASM algorithm after 3 iterations with a relative error of $0.074 \%$ ), are $Z_{o}=104.10 \Omega, k l=$ $22.50^{\circ}$, and $C_{l s}=1.73 \mathrm{pF}$. The response of the schematic, depicted in Fig. 6, indicates that the target specifications at the schematic level are satisfied to a very good approximation also for this second example. The resulting electrical length, Bloch impedance and slow-wave factor of the cell after convergence are found to be $Z_{B}=49.97 \Omega, \phi_{\text {cell }}=45.01^{\circ}$, and $s w r=0.50$, respectively. The application of the second ASM algorithm has provided the layout depicted in Fig. 7 (the same substrate has been used). The electromagnetic response is also depicted in Fig. 6 , and reveals that the target specifications are also satisfied to a very good approximation at the design frequency. The measured response is also in good agreement at the design frequency, 
except for the previously mentioned discrepancies. The characteristic impedance, inferred from the measured S-parameters, is noisy below $0.4 \mathrm{GHz}$ and is not shown.

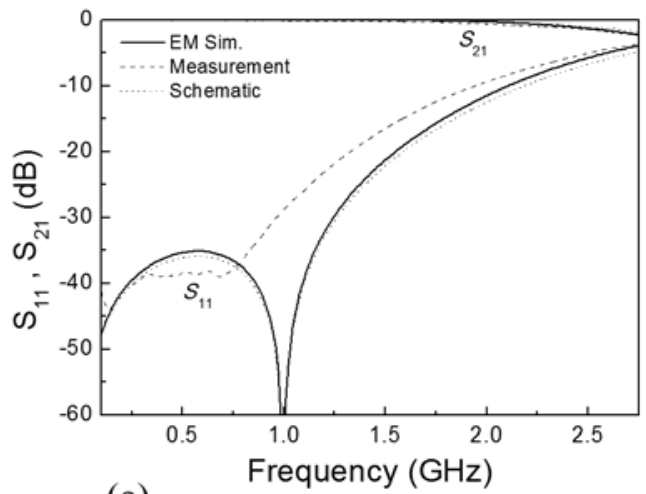

(a)

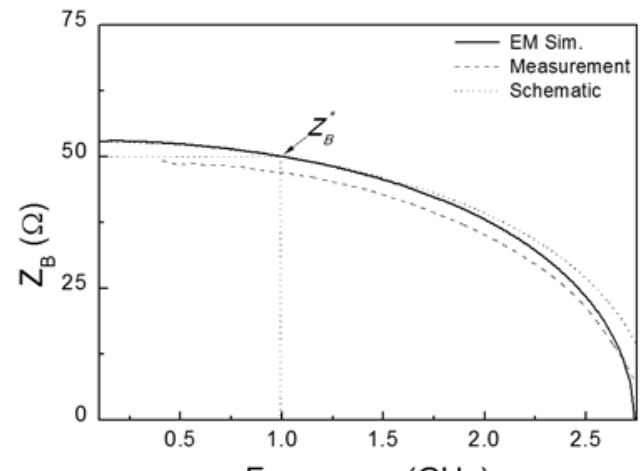

(b)

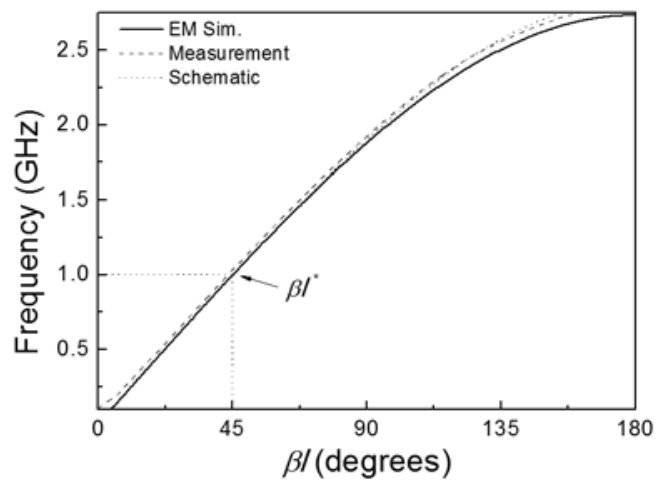

(c)

Fig. 6. Response of the cell considered in example 2. (a) Insertion and return loss, (b) characteristic impedance, and (c) electrical length. 


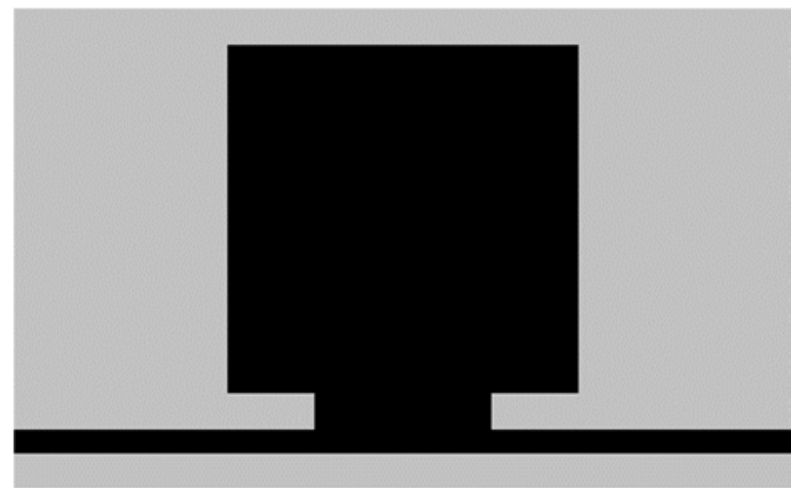

(a)

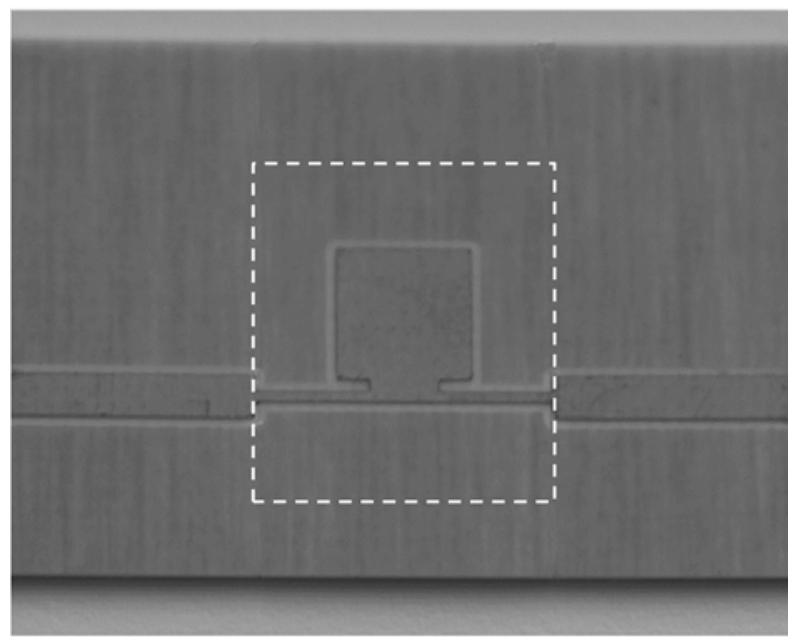

(b)

Fig. 7. Layout (a) and photograph (b) of the synthesized cell of example 2. Dimensions are: $l=12.73$ $\mathrm{mm}, l_{p}=5.75 \mathrm{~mm}, W_{\text {acc }}=2.87 \mathrm{~mm}\left(0.5 l_{p}\right)$, $W_{\text {host }}=0.41 \mathrm{~mm}$.

\section{Conclusions}

In summary, we have developed an optimization algorithm for the synthesis of slow-wave transmission lines consisting on microstrip lines loaded with patch capacitors. The algorithm, based on the so-called aggressive space mapping (ASM), uses quasi-Newton type iteration, and has been divided in two steps. The first ASM determines the schematic providing the target specifications (optimum schematic), that is, the slow-wave ratio, the Bloch impedance and the per-unit-cell electrical length; the second step, with two independent ASM sub-processes, provides the layout of the slow-wave structure from the optimum cell schematic. Two illustrative synthesis examples have been provided to demonstrate the potential of the proposed synthesis approach. The main relevant aspect of the proposed two-step ASM algorithm is that the layout of the structures is determined from the target specifications in a completely unattended scheme.

\section{Acknowledgements}

This work has been supported by MINECO-Spain (projects TEC2010-17512 METATRANSFER, TEC2010-21520-C04-01, TEC2013-47037-C5-1-R, CONSOLIDER EMET CSD2008-00066, TEC2013-40600-R and TEC2013-49221-EXP), Generalitat de Catalunya (project 2014SGR-157), and Institució Catalana de Recerca i Estudis Avançats (who 
has awarded Ferran Martín). Marco Orellana acknowledges the support of the Universidad de Costa Rica, MICITT and CONICIT to study at the Univesitat Autònoma de Barcelona. 


\section{References}

1. F. Martín, J. Bonache, M. Durán-Sindreu, J. Naqui, F. Paredes, G. Zamora, “Artificial transmission lines”, in Wiley Encyclopedia of Electrical and Electronic Engineering, July 2012.

2. A. Görür, “A novel coplanar slow-wave structure”, IEEE Microwave and Guided Wave Letters, vol. 4, no. 3, pp. 86-88, March 1994.

3. J. Sor, Y. Qian, and T. Itoh, "Miniature low loss CPW periodic structures for filter applications", IEEE Transactions on Microwave Theory and Techniques, vol 49, pp. 2336-2341, 2001.

4. S.G. Mao and M.Y. Chen, "A novel periodic electromagnetic bandgap structure for finite-width conductor-backed coplanar waveguides”, IEEE Microwave and Wireless Components Lett., vol 11, pp. 261-263, 2001.

5. F. Martín, F. Falcone, J. Bonache, M.A.G. Laso, T. Lopetegi, M. Sorolla, “ New CPW low pass filter based on a slow wave structure”, Microwave and Optical Technology Lett., vol. 38, pp. 190-193, 2003.

6. J. García-García J. Bonache and F. Martín, “ Application of electromagnetic bandgaps (EBGs) to the design of ultra wide band pass filters (UWBPFs) with good out-of-band performance”, IEEE Transactions on Microwave Theory and Techniques, vol. 54, pp. 4136 - 4140 , Dec. 2006.

7. J.W. Bandler, R.M. Biernacki, S.H. Chen, P.A. Grobelny and R.H. Hemmers, "Space mapping technique for electromagnetic optimization”, IEEE Transactions on Microwave Theory and Techniques, vol. 42, pp. 2536-2544, Dec. 1994.

8. J.W. Bandler, R.M. Biernacki, S.H. Chen, R.H. Hemmers, and K. Madsen, "Electromagnetic optimization exploiting aggressive space mapping", IEEE Transactions on Microwave Theory and Techniques, vol. 43, pp. 2874-25442882, Dec. 1995.

9. S. Koziel, Q.S. Cheng, J.W. Bandler, “Space mapping”, IEEE Microwave Magazine, vol. 9, pp. 105-122, Dec. 2008.

10. L. J. Rogla, J. E. Rayas-Sanchez, V. E. Boria, and J. Carbonell, "EM-Based Space Mapping Optimization of Left-handed Coplanar Waveguide Filters with Split Ring Resonators", IEEE MTT-S International Microwave Symposium Digest, pp. 111-114, 38 June 2007.

11. P. J. Bradley, "Robust surrogate-based optimisation of planar metamaterial structures", IEEE International Conference on Wireless Information Technology and Systems, pp. 1-4, 11-16 Nov. 2012.

12. P. J. Bradley, "Quasi-newton model-trust region approach to surrogate-based optimisation of planar metamaterial structures", Progress In Electromagnetics Research B, vol. 47, pp. 1-17, 2013.

13. J. Selga, A. Rodríguez, V.E. Boria, and F. Martín, "Synthesis of split rings based artificial transmission lines through a new two-step, fast converging, and robust aggressive space mapping (ASM) algorithm”, IEEE Transactions on Microwave Theory and Techniques, vol. 61(6), pp. 2295-2308, June 2013. 
14. J. Selga, A. Rodríguez, J. Naqui, M. Durán-Sindreu, V.E. Boria and F. Martín, "Application of aggressive space mapping (ASM) to the efficient synthesis of stepped impedance resonators (SIRs)", European Microwave Conference, Nuremberg (Germany), 2013.

15. J. Selga, M. Sans, A. Rodríguez, J. Bonache, V. Boria, F. Martín, “Automated synthesis of planar wideband bandpass filters based on stepped impedance resonators (SIRs) and shunt stubs through aggressive space mapping (ASM)”, IEEE MTT-S Int. Microwave Symp., June 2014, Tampa, FL (USA).

16. M. Sans, J. Selga, A. Rodríguez, J. Bonache, V.E. Boria, and F. Martín, "Design of planar wideband bandpass filters from specifications using a two-step aggressive space mapping (ASM) optimization algorithm”, IEEE Transactions on Microwave Theory and Techniques, vol. 62, pp. 3341-3350, Dec. 2014.

17. D.M. Pozar, Microwave Engineering, Addison Wesley, 1990.

18. C. G. Broyden, "A Class of Methods for Solving Nonlinear Simultaneous Equations", Mathematics of Computation, vol. 19, no. 92, pp. 577-593, Oct. 1965. 


\section{Biographies}

Marco Orellana was born in San José, Costa Rica, in 1985. He earned a Licentiate degree in Electrical Engineering from the Universidad de Costa Rica (UCR) in 2009. In 2011 obtained the Master degree in Micro and Nanoelectronics Engineering at the Universitat Autònoma de Barcelona with the support of the Erasmus Mundus Programme and the UCR. He has been instructor at the School of Electrical Engineering at UCR. He is currently working on his Doctoral Thesis at the Universitat Autònoma de Barcelona focused on the automation of metamaterial-based structures design.

Jordi Selga was born in Barcelona, Spain, in 1982. He received the Telecommunications Engineering - Electronic Systems diploma in 2006, the Electronics Engineering degree in 2008 and the PhD degree in Electronics Engineering in 2013 from the Universitat Autònoma de Barcelona (UAB), Barcelona, Spain. Since 2008 is member of CIMITEC-UAB, a research centre on Metamaterials supported by TECNIO (Catalan Government). He was holder of a national research fellowship from the Formación de Profesorado Universitario Program of the Education and Science Ministry (Reference AP2008-4707). He is currently working in subjects related to metamaterials, CAD design of microwave devices, EM optimization methods and automated synthesis of planar microwave components at the UAB.

Marc Sans was born in Terrassa (Barcelona), Spain, in 1982. He received the B.S. Degree in Telecommunications Engineering - Electronic Systems in 2006, the M.S. Degree in Telecommunications Engineering in 2008 and the M.S. Degree in Electronics Engineering in 2013 from the Universitat Autònoma de Barcelona (UAB). In 2008 he started his professional career as a RF Engineer at Sony-FTVE developing the RF stage of TV receivers. In 2010 he moved to Mier Comunicaciones S.A. to carry out the design of passive and active devices for VHF-UHF broadcasting units. Since 2014 he is working towards the PhD Degree at CIMITEC$\mathrm{UAB}$ in the synthesis of microwave devices based on EM optimization techniques.

Ana Rodriguez was born in Lugo, Spain. She received the Telecommunications Engineering degree from the Universidade de Vigo (UV), Spain, in 2008. As a student, she participated in the Erasmus exchange program, developing the Master Thesis at the University of Oulu, Finland. Since the end of 2008, she has joined the Institute of Telecommunications and Multimedia Applications (iTEAM), which is part of the scientific park at the Universitat Politècnica de València (UPV), Spain. She obtained "Master en Tecnología, Sistemas y Redes de Comunicaciones" in 2010 from UPV, and currently is on the way of pursuing Ph.D. degree. Her main research interests include CAD design of microwave devices, EM optimization methods and metamaterials.

Vicente E. Boria was born in Valencia, Spain, on May 18, 1970. He received his "Ingeniero de Telecomunicación" degree (with first-class honors) and the "Doctor Ingeniero de Telecomunicación” degree from the Universidad Politécnica de Valencia, Valencia, Spain, in 1993 and 1997, respectively. In 1993 he joined the "Departamento de Comunicaciones", Universidad Politécnica de Valencia, where he has been Full Professor since 2003. In 1995 and 1996, he was holding a Spanish Trainee position with the European Space Research and Technology Centre, European Space Agency (ESTEC-ESA), Noordwijk, The Netherlands, where he was involved in the area of EM analysis and design of passive waveguide devices. He has authored or co-authored 7 chapters in technical textbooks, 75 papers in refereed international technical journals, and over 150 papers in international conference proceedings. 
His current research interests are focused on the analysis and automated design of passive components, left-handed and periodic structures, as well as on the simulation and measurement of power effects in passive waveguide systems.

Dr. Boria has been a member of the IEEE Microwave Theory and Techniques Society (IEEE MTT-S) and the IEEE Antennas and Propagation Society (IEEE AP-S) since 1992. He is reviewer of the IEEE Transactions on Microwave Theory and Techniques, Proceeding of the IET (Microwaves, Antennas and Propagation) and IET Electronics Letters. Since 2013, he serves as Associate Editor of IEEE Microwave and Wireless Components Letters. He is also a member of the Technical Committees of the IEEE-MTT International Microwave Symposium and of the European Microwave Conference.

Ferran Martín was born in Barakaldo (Vizcaya), Spain in 1965. He received the B.S. Degree in Physics from the Universitat Autònoma de Barcelona (UAB) in 1988 and the $\mathrm{PhD}$ degree in 1992. From 1994 up to 2006 he was Associate Professor in Electronics at the Departament d'Enginyeria Electrònica (Universitat Autònoma de Barcelona), and since 2007 he is Full Professor of Electronics. In recent years, he has been involved in different research activities including modelling and simulation of electron devices for high frequency applications, millimeter wave and $\mathrm{THz}$ generation systems, and the application of electromagnetic bandgaps to microwave and millimeter wave circuits. He is now very active in the field of metamaterials and their application to the miniaturization and optimization of microwave circuits and antennas. He is the head of the Microwave Engineering, Metamaterials and Antennas Group (GEMMA Group) at UAB, and director of CIMITEC, a research Center on Metamaterials supported by TECNIO (Generalitat de Catalunya). He has organized several international events related to metamaterials, including Workshops at the IEEE International Microwave Symposium (years 2005 and 2007) and European Microwave Conference (2009), and the Fifth International Congress on Advanced Electromagnetic Materials in Microwaves and Optics (Metamaterials 2011), where he has acted as chair of the Local Organizing Committee. He has acted as Guest Editor for three Special Issues on Metamaterials in three International Journals. He has authored and co-authored over 450 technical conference, letter, journal papers and book chapters, he is co-author of the book on Metamaterials entitled Metamaterials with Negative Parameters: Theory, Design and Microwave Applications (John Wiley \& Sons Inc.), and he has generated 14 PhDs. Ferran Martín has filed several patents on metamaterials and has headed several Development Contracts.

Prof. Martín is a member of the IEEE Microwave Theory and Techniques Society (IEEE MTTS). He is reviewer of the IEEE Transactions on Microwave Theory and Techniques and IEEE Microwave and Wireless Components Letters, among many other journals, and he serves as member of the Editorial Board of IET Microwaves, Antennas and Propagation and International Journal of RF and Microwave Computer-Aided Engineering. He is also a member of the Technical Committees of the European Microwave Conference (EuMC) and International Congress on Advanced Electromagnetic Materials in Microwaves and Optics (Metamaterials). Among his distinctions, Ferran Martín has received the 2006 Duran Farell Prize for Technological Research, he holds the Parc de Recerca UAB - Santander Technology Transfer Chair, and he has been the recipient of two ICREA ACADEMIA Awards (calls 2008 and 2013). 